\title{
IMUNOEXPRESSÃO DO C-ERBB-2 NAS LESÕES EPITELIAIS PROLIFERATIVAS INTRADUCTAIS DA MAMA DE MULHERES
}

\author{
Agliberto Barbosa de Oliveira*, laurival Antônio De luca, Grigna Teixeira Carvalho, \\ Victor Eduardo Arua Arias, lídia Raquel de Carvalho, Maria do Carmo Assunção \\ Trabalho realizado no departamento de Ginecologia e Obstetrícia da Faculdade \\ de Medicina de Botucatu - UNESP, Botucatu, SP.
}

RESUMO - OBjetivos. Alterações genéticas são relacionadas à gênese e progressão do câncer. Neoplasias de vários órgãos expressam o oncogene c-erbB-2. Nas proliferações intraductais da mama tem sido avaliado como fator de risco para o desenvolvimento de câncer. Foram avaliadas a imunoexpressão do c-erbB-2 em lesões epiteliais proliferativas intraductais e as possíveis correlações com características anatomopatológicas do carcinoma ductal "in situ" (CDIS).

MÉtodos. Foi utilizado material de arquivo, amostras teciduais fixadas em formalina e incluídas em blocos de parafina de $\mathbf{8 8}$ mulheres. Destas, 5 I com CDIS e 37 com hiperplasia ductal sem atipias (HDT). A idade variou de 35 a 76 anos. Revisados todos os casos, verificou-se: 0 grau nuclear, a presença de necrose, o subtipo histológico predominante e sua extensão. Obteve-se material suficiente para o estudo imunohistoquímico do c-erbB- 2 de 84 sujeitos do estudo.
Resultados. Não foi observada a expressão do oncogene nas hiperplasias sem atipias e nos tecidos adjacentes a todas amostras teciduais. A expressão do c-erbB-2 foi verificada em nove ( $19,1 \%)$ dos CDIS $(p=0,000 I)$. A imunoexpressão não se relacionou à extensão das lesões. A imunoexpressão do c-erbB-2 no CDIS correlacionou-se com subtipo histológico $(p=0,019)$, com a presença de necrose $(p=0,0066)$, com o grau nuclear $(p=0,0084)$ e com a Classificação de Van Nuys $(p=0,039)$.

Conclusōes. A expressão do c-erbB-2 foi estatisticamente significante nas lesões proliferativas de risco (CDIS) e correlacionou-se com características histopatológicas: alto grau nuclear, presença de necrose, subtipo comedo. Não houve expressão nas hiperplasias sem atipias e tecidos adjacentes.

UNITERMOS: Carcinoma ductal "in situ”. Lesões proliferativas intraductais. C-erbB-2. Prognóstico. Câncer de mama.

\section{INTRODUÇÃo}

Graças à evolução tecnológica dos meios de diagnóstico precoce, os diagnósticos de lesões precursoras e invasivas subclínicas da mama aumentaram consideravelmente. Nos EUA, nos últimos 20 anos, a incidência do câncer de mama (CM) aumentou 50\% e a das lesões pré invasivas em 235\% 1,2.

Broders (1932) definiu carcinoma "in situ" como "... a condição na qual as células malignas e suas descendentes encontram-se no local ocupado pelas suas ancestrais antes que estas sofressem transformação maligna, e não migraram além da membrana basal"

No passado, o carcinoma ductal "in situ" (CDIS) tinha menor incidência e habitualmente as lesões eram palpáveis. Considerado como o primeiro degrau na progressão para o carcinoma da mama ${ }^{4}$, seu tratamento rotineiramente consistia em mastectomia. Sabe-se,

*Correspondência:

Rua Frederico Abranches, $389-\mathrm{Cj} .52$

CEP: 01225-001 - São Paulo - SP atualmente, que não é entidade biologicamente homogênea, mas espectro de lesões com diferenças clínicas, histopatológicas e biomoleculares, que determinam sua evolução e os diversos procedimentos terapêuticos ${ }^{5-8}$.

O tratamento deve adequar-se ao risco evolutivo de cada caso. Quanto melhor se conhecer os fatores anatomopatológicos e os biomoleculares, mais racional será a orientação terapêutica.

Como fatores prognósticos, anatomopatológicos, de evolução das neoplasias intraductais são considerados: o tamanho da lesão, o grau nuclear, o subtipo histológico, a presença de necrose e a extensão livre das margens cirúrgicas. Nas lesões invasivas, há indícios de que os oncogenes p2I e c-erb-2 são fatores prognósticos independentes. A freqüência de recidivas se associa com a expressão de p2I maior que $15 \%$ e positividade da expressão do c-erbB-2. Estas observações, entretanto, não permitem a inclusão destes fatores no estadiamento clínico dos tumores invasivos e com maior razão nas lesões pré-invasivas?

$O$ gene neu, identificado em estudo de células de cultura NIH3T3 transfectadas com DNA de neuroblastomas induzidos com etilnitrosuréia $^{10}$, localiza-se no cromossomo 17 q 21 e codifica a síntese de proteína de 185 kD (kilodaltons) denominada p $185^{\text {erb }}$, que é semelhante ao receptor de fator de crescimento epidérmico. O c-erbB-2 é amplificado ou superexpresso em 20\% a $30 \%$ dos carcinomas ductais e em $40 \%$ a $70 \%$ das lesões intraductais $7,11,12,13$. Sua imunoexpressão foi avaliada em pequenas séries de hiperplasias ductais, com e sem atipias $11,14,15$ e nas neoplasias intraductais aos diferentes subtipos histológicos ${ }^{14-17}$, à presença de necrose ${ }^{19,20}$, aos diferentes graus nucleares ${ }^{13,19}$; à apoptose ${ }^{21}$ e à recorrência ${ }^{22}$.

Neste trabalho evitamos a designação carcinoma ductal "in situ", substituindo-a por nomenclatura mais atual e condizente com os aspectos anatomopatológicos e risco-evolutivos: lesões epiteliais proliferativas intraductais de risco (LEPIS $)^{23}$.

A carcinogênese abrange múltiplas etapas. É possível que o número de alterações moleculares aumente durante a fase de progres- 
são, mas algumas estão presentes nas fases mais iniciais. Há indícios de que a sua identificação tem importância para o esclarecimento da patogênese das lesões intraductais e influência na identificação de seu risco evolutivo.

Os objetivos deste estudo foram avaliar a imunoexpressão do c-erbB-2 em lesões epiteliais proliferativas intraductais da mama e nos ductos normais adjacentes; e averiguar possíveis correlações entre a expressão imunohistoquímica do oncogene c-erbB-2 com outras características anatomopatológicas.

\section{Métodos}

Foi realizado estudo retrospectivo com material arquivado de série pessoal de 51 neoplasias ductais "in situ" tratadas entre 1982 e 2000. Como critérios de inclusão foram considerados: neoplasia de crescimento exclusivamente intraductal e tratamento cirúrgico completo confirmando a exérese de toda a lesão. O tratamento cirúrgico consistiu em: mastectomia (nove) e ressecções segmentares (42). $O$ estudo estatístico prévio considerou adequado para comparação um grupo de 37 pacientes com diagnóstico de hiperplasia ductal sem atipias. Todas as mulheres com HDT haviam sido submetidas à ressecção cirúr gica de lesões mamárias, que foram totalmente extirpadas, sendo demonstradas suas margens livres.

O protocolo desta pesquisa foi apresentado ao Comitê de Ética em Pesquisa - Faculdade de Medicina de Botucatu - Universidade Estadual Paulista, tendo recebido parecer de aprovação em 2 de outubro de 2000. Não foi recomendado o Termo de Consentimento Livre e Esclarecido.

\section{Estudo anatomopatológico}

Foi estudado material fixado em formalina e incluído em parafina de 88 mulheres, portadoras de lesões proliferativas intraductais da mama de diverso risco evolutivo. Delas, 51 tinham neoplasias ductais "in situ" (grupo A), e 37 (grupo B) apresentavam hiperplasia ductal sem atipias (HDT).

Os laudos anatomopatológicos e a totalidade das lâminas disponíveis foram revistos para confirmação diagnóstica obtida em todos os casos, e subseqüente inclusão em cada um dos grupos. Foram analisados 1572 cortes histológicos (média de 30,82 por paciente) no grupo A e 684 (média de 18,48 por paciente) no grupo $B$.

O tamanho das lesões foi aferido pelo maior diâmetro. As medidas foram obtidas por mensuração direta nas lesões macroscopicamente identificáveis, ou mensuração pela microscopia nas incluídas totalmente em um único corte histológico, ou pela soma dos fragmentos com lesão microscópica.

Não foi possível a realização do estudo imunohistoquímico em quatro casos do grupo $\mathrm{A}$, devido à quantidade insuficiente de material.

As lesões proliferativas foram classificadas separadamente por duas patologistas co-autoras do trabalho. Havendo discordância, o diagnóstico definitivo foi obtido por consenso entre ambas. O diagnóstico de hiperplasia ductal, simples baseou-se em critérios amplamente conhecidos ${ }^{24}$. Nos casos de neoplasia intraductal, levou-se em consideração: o grau nuclear, presença ou ausência de necrose, arquitetura dominante e agrupados segundo a Classificcação "Van Nuys" 25 . A classificação nuclear estribou-se nos critérios de Lagios (1990)26:

Grau nuclear I (baixo grau): núcleos com diâmetro comparável ao de uma a uma vez e meia o de uma hemácia adjacente, cromatina difusa e nucléolos inaparentes.

Grau nuclear 2 (intermediário): núcleos com diâmetro de uma e meia a duas vezes o de uma hemácia adjacente, cromatina grosseira, nucléolos pouco freqüentes.

Grau nuclear 3 (alto grau): núcleos com diâmetro duas vezes e meia maior que o de uma hemácia adjacente, apresentando cromatina irregular e um ou mais nucléolos visíveis.

Considerou-se como necrose a presença de células mortas e debris com cinco ou mais núcleos cariopicnóticos na luz dos ductos ${ }^{27}$.

Quanto ao padrão arquitetural, nos casos que exibiam mais de um padrão de crescimento neoplásico, considerou-se como dominante o observado em mais de $50 \%$ da lesão, e como subtipos: comedo, sólido, cribriforme, micropapilar, papilar e de células claras.

\section{Estudo imunohistoquímico}

A imunoexpressão do oncogene c-erbB-2 foi avaliada nas lesões e em seus tecidos adjacentes. Para tal, utilizou-se o método da estreptavidina-biotina-peroxidase com anticorpo policlonal, por técnica descrita previamente ${ }^{28}$. Brevemente, os cortes histológicos de $4 \mathrm{~m}$ de espessura, montados em lâminas silanizadas (3-aminopropiltrietoxisilano, marca Sigma, código A3648) foram desparafinados e rehidratados e submetidos à recuperação antigênica em forno micro-ondas (dois ciclos de nove minutos à potência de $1400 \mathrm{~W}$ em tampão citrato $10 \mathrm{mM}, \mathrm{pH} 6,0)$. O bloqueio da peroxidase endógena foi alcançado lavando-se as lâminas com água oxigenada a 3\%. O anticorpo primário utili zado foi o antioncoproteína c-erbB-2 (policlonal, Dako, código A0485) diluído a 1/200. Carcinoma mamário com positividade previamente conhecida oncoproteína c-erbB-2 foi utilizado como controle positivo para essas reações. O controle negativo foi obtido substituindo-se 0 anticorpo primário por solução salina tamponada.

A imunoexpressão do c-erbB-2 foi confirmada com a coloração das mem branas celulares em cor castanha. Os resultados foram analisados semiquantitativamente utilizando-se os seguintes parâmetros:

(-): nenhuma célula positiva ou positividade em menos de $10 \%$ das células

$(+)$ : imunorreatividade de membrana, fraca e irregular em pelo menos $10 \%$ das células.

$(++)$ : imunorreatividade de membrana, de intensidade fraca a moderada, porém regular em mais de 10\% das células.

$(+++)$ : imunorreatividade de membrana, com forte intensidade, em pelo menos $10 \%$ das células.

\section{Análise estatística}

Foi avaliada a imunoexpresão do oncogene c-erbB-2 nas hiperplasias e nas neoplasias intraductais. E nas lesões do grupo $A$, as associações com as variáveis: tamanho, tipo histológico dominante, grau nuclear, necrose, Classificação de Van Nuys.

Foram aplicados os testes Exato de Fisher e Proporções ${ }^{29}$.

O nível de significância adotado foi de $5 \%$ ou seja, valores de $p<0,05$ foram considerados estatisticamente significantes. 

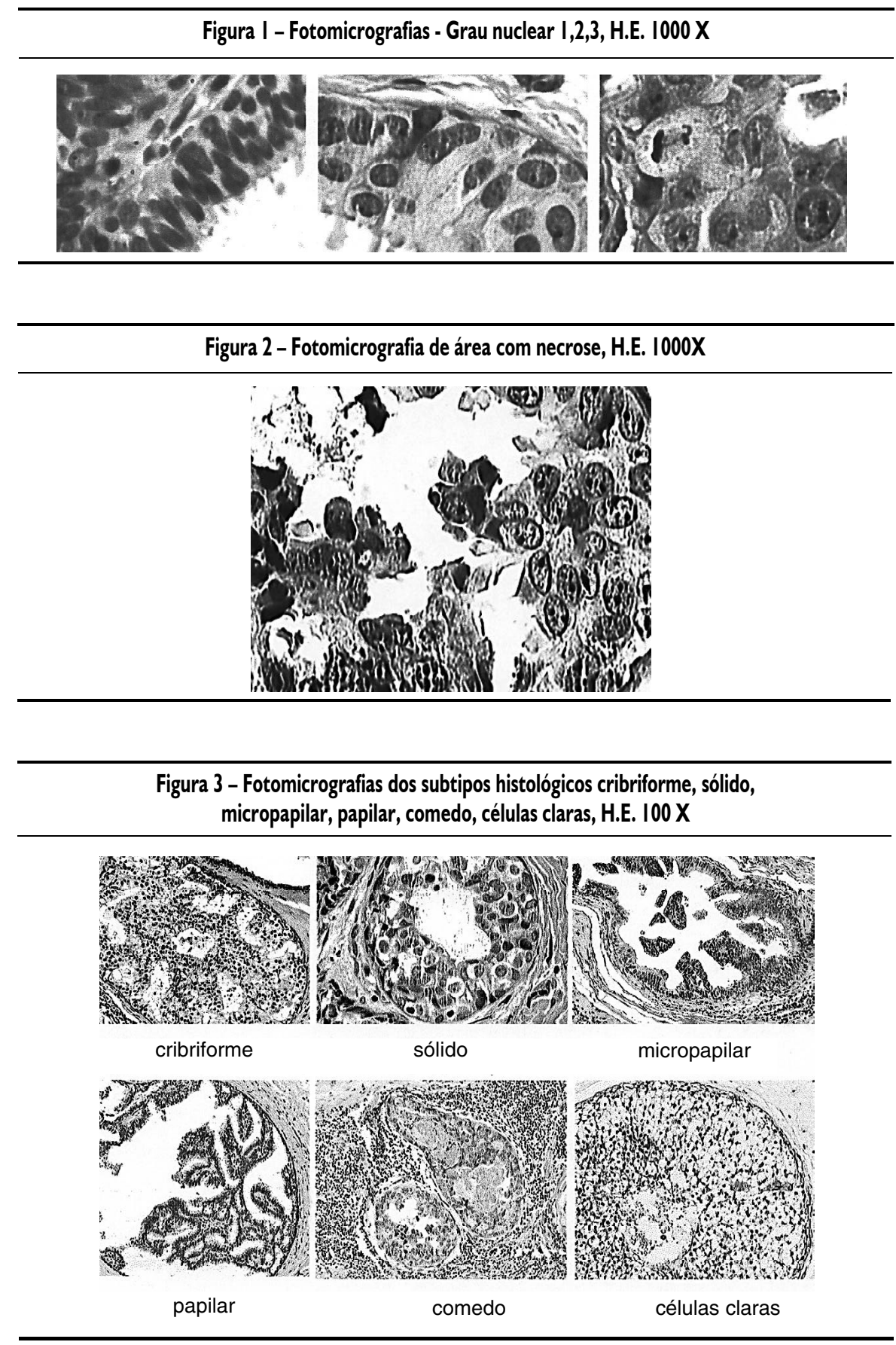

\section{Resultados}

A idade das pacientes variou entre $35 \mathrm{e}$ 76 anos no grupo $A$ (média $=53,86$ anos), no grupo $B$, entre 39 e 73 anos (média= 51,43 anos).

O estudo anatomopatológico foi completo nos 88 sujeitos do estudo. Entretanto, a técnica imunohistoquímica do c-erbB-2 não pôde ser realizada em quatro amostras do grupo $A$, devido à exigüidade dos materiais arquivados. Desta forma, a imunoexpressão foi avaliada em 47 amostras teciduais do grupo A (CDIS) e em 37 do grupo B (HDT).

As freqüências absolutas e relativas dos tipos histológicos dominantes foram: cribriforme $18(35,2 \%)$, comedo $15(29,4 \%)$, micropapilar 10 (19,6\%), sólido 7(13,7\%), células claras I ( I,9\%).
Foi constatada a presença de necrose em 28 (54,9\%) das pacientes com lesões epiteliais proliferativas de maior risco. A presença de necrose faz parte da definição do subtipo comedo, portanto observada em todos os casos deste subtipo e também na única lesão do padrão células claras. Nos tipos micropapilar, sólido e cribriforme foi observada em 10\%, 28,6\% e $50 \%$ dos casos, respectivamente. ( $p=0,000005$ teste Exato de Fisher)

As freqüências dos diversos graus nucleares foram: grau $3(39,3 \%)$, grau $2(33,3 \%)$ e grau I $(27,4 \%)$.

A necrose foi observada em 19 (95\%) das lesões grau 3, e em nove (52,9\%) das de grau 2 e ausente na totalidade das 14 lesões de grau 1.

Nas 22 lesões do grupo I da Classificação de Van Nuys, foram observados os seguintes padrões arquiteturais: micro papilar em nove $(40,9 \%)$, cribriforme em nove (40,9\%), e sólido em quatro (I8,2\%); no grupo Van Nuys 2, a maior freqüência foi do subtipo cribriforme cinco lesões $(55,5 \%)$, seguido por dois padrão comedo $(22,2 \%)$, um padrão sólido e um de células claras $(\mathrm{I}, \mid \mathrm{l} \%)$. Entre as classificadas como Van Nuys 3, o subtipo comedo foi notado em I 3 (65\%), o cribriforme em quatro (20\%), o sólido em dois ( $10 \%)$ e o micropapilar em um $(5 \%)(p=0,000007)$.

A imunoexpressão do c-erbB-2 foi avaliada nas lesões proliferativas, e nos tecidos normais adjacentes. No grupo A, lesões epiteliais proliferativas intraductais de maior grau e maior risco também foram relacionadas às suas características histopatológicas.

A imunoexpressão do c-erbB-2 foi verificada em nove $(19,1 \%)$ das neoplasias intraductais e ausente na totalidade das hiperplasias ductais sem atipias, bem como nos tecidos adjacentes de ambos grupos, $p=0,000 \mathrm{I}$. A intensidade da coloração foi de $(+)$ em três pacientes, $(++)$ em uma e $(+++)$ em cinco.

Para os cálculos estatísticos a expressão do c-erbB-2 foi considerada positiva ou negativa, não tendo sido feita estratificação pela intensidade da reação.

A imunoexpressão do c-erbB-2 em relação ao tamanho das neoplasias "in situ" não apresentou correlação estatística significante $p=0,945$ (Tabela I). 


\section{Figura 4 - Fotomicrografias da imunoexpressão do c-erbB-2} $(+),(++),(+++)$, H.E. $400 X$

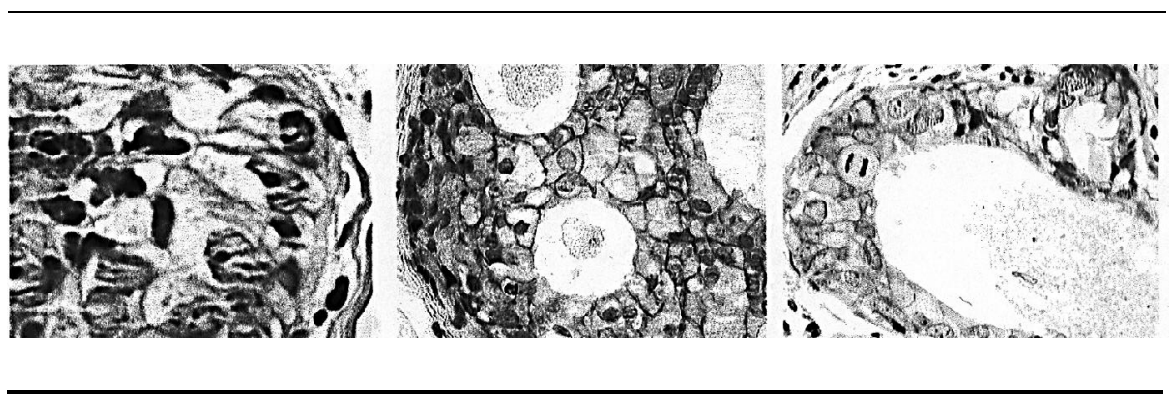

Tabela I - Imunoexpressão do c-erbB-2 no carcinoma ductal "in situ" em relação ao tamanho, ao tipo histológico, à necrose, ao grau nuclear e à classificação de Van Nuys

\begin{tabular}{|c|c|c|c|c|}
\hline \multirow[b]{3}{*}{ Tamanho (mm } & \multicolumn{4}{|c|}{ c-erbB-2 } \\
\hline & $\begin{array}{c}\text { positivo } \\
\mathrm{n}(\%)\end{array}$ & $\begin{array}{c}\text { negativo } \\
\mathrm{n}(\%)\end{array}$ & $\begin{array}{l}\text { Total } \\
n(\%)\end{array}$ & $p$ \\
\hline & & & & \\
\hline$<6$ & $I(\mid 0,0)$ & $9(90,0)$ & $10(100,0)$ & \\
\hline $6<11$ & $\mid(\mid 6,6)$ & $5(83,4)$ & $6(100,0)$ & \\
\hline||$<2 \mid$ & $1(25,0) 0$ & $3(75,0)$ & $4(100,0)$ & \\
\hline $21<50$ & $5(22,7)$ & $17(77,3)$ & $22(100,0)$ & \\
\hline$\geq 50$ & $\mid(20,0)$ & $4(80,0)$ & $5(100,0)$ & $0,945^{(1)}$ \\
\hline \multicolumn{5}{|c|}{ Tipo histológico } \\
\hline Células claras & $0(0,00)$ & I(100,0) & $\mid(100,0)$ & \\
\hline Comedo & $7(46,7)$ & $8(53,3)$ & $15(100,0)$ & \\
\hline Cribriforme & $\mid(6,2)$ & $15(93,8)$ & $16(100,0)$ & \\
\hline Micropapilar & $0(0,0)$ & $9(100,0)$ & $9(100,0)$ & \\
\hline Sólido & $\mid(\mid 6,7)$ & $5(83,3)$ & $6(100,0)$ & $0,019 *(2)$ \\
\hline
\end{tabular}

\section{Necrose}

Presente

Ausente

$9(32,1)$

$0(0,0)$

$19(67,9)$

$19(100,0)$

$28(100,0)$

$19(100,0)$

$0,0066 *(3)$

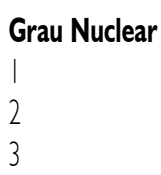

$10(100,0)$
$16(94,1)$
$12(60,0)$

$10(100,0)$

$17(100,0)$

$20(100,0)$

\section{Van Nuys}

\begin{tabular}{ccccc}
1 & $0(0,0)$ & $18(100,0)$ & $18(100,0)$ & \\
2 & $1(11,1)$ & $8(88,9)$ & $9(100,0)$ & \\
3 & $8(40,0)$ & $12(60,0)$ & $20(100,0)$ & $0,039 *(5)$ \\
\hline
\end{tabular}

* estatisticamente significante $p<0,05$

(I) Teste Proporções

(2) (3) (4) (5) Teste Exato de Fisher

Em relação aos diferentes subtipos, sete $(46,7 \%)$ das lesões comedo, um $(16,7 \%)$ do tipo sólido e um (6,2\%) do tipo cribriforme foram positivas no estudo imunohistoquímico do c-erbB-2. Não foi observada expressão do oncogene nos subtipos micropapilar e de células claras $p=0,019 *$ (Tabela I). Entre os 28 casos que exibiam necrose, nove $(32,2 \%)$ eram c-erbB-2 positivos. Não se notou expressão do oncogene em nenhum dos casos sem necrose $p=0,0066 *$ (Tabela I).

$\mathrm{Na}$ correlação entre grau nuclear e c-erbB-2, verificou-se a expressão positiva do c-erbB-2 em oito (40\%) das lesões de grau 3, um $(5,9 \%)$ das de grau 2 e em nenhuma do grau I $p=0,0084$ (Tabela I).

Estudando-se a associação entre a imunoexpressão do c-erbB-2 e as lesões classificadas segundo Van Nuys, observouse que nenhuma das lesões Van Nuys I exprimia reação imunohistoquímica; nas classificadas como Van Nuys 2 e 3 a reação foi positiva em um (II, I\%) e oito (40\%), respectivamente $p=0,0039$ (Tabela I).

\section{Discussão}

O aumento do número de casos diagnosticados de neoplasias intraductais suscitou questionamentos sobre sua história natural, e o maior ou menor risco de evolução para câncer 2 2,9,30,31.

Existem evidências mostrando que as lesões "in situ" são precursoras do câncer de mama. Entretanto, pairam dúvidas a respeito do potencial evolutivo das formas mais iniciais de proliferação intraductal. Ainda que os aspectos morfológicos sejam sugestivos de evolução a partir da hiperplasia ductal sem atipias para hiperplasia com atipias e para formas mais anaplásicas inadequadamente denominadas de carcinoma "in situ"23.

Nesta investigação não se constatou expressão do oncogene nos casos de hiperplasias ductais sem atipias e nos seus tecidos adjacentes, coincidindo com resultados publicados por outros autores que utilizaram a mesma metodologia ${ }^{10}$, corroborando a hipótese de que a imunoexpressão do oncogene é evento precoce no processo da carcinogenese.

A expressão imunohistoquímica do c-erbB-2 nas neoplasias intraductais foi avaliada em várias séries, oscilando os resultados 
positivos entre $27 \%$ e $61 \% \%^{11,19,32,33,34,35}$. Atribuem-se as variações dos resultados às metodologias diversas, com anticorpos e diluições diferentes, e às pequenas amostras estudadas. Verificou-se, nesse estudo, a positividade da expressão do c-erbB-2 em nove (19,1\%) das amostras teciduais, e sua ausência na totalidade dos tecidos adjacentes.

O tamanho da lesão, que é reconhe cido como fator prognóstico anatomo patológico de recidiva, não foi relacionado à imunoexpressão do c-erbB-2 em outras séries $^{19}$, coincidindo com o observado nessa série, onde as lesões positivas para o c-erbB-2 distribuíam-se de forma eqüitativa em todas as faixas de tamanho, não confirmando significância estatística na correlação entre a expressão do oncogene e o tamanho da lesão.

As lesões do subtipo histológico comedo foram relacionadas à recidiva e progressão para invasão $11,17,18,36,37,38$, e associadas à maior expressão do c-erbB-2 $11,16,17,36-40$.

Essas observações coincidem com diversas publicações em relação à predominância da expressão do oncogene no subtipo comedo. Nesta casuística, sete $(77,7 \%)$ das amostras que exprimiam reação positiva para o c-erbB-2 eram de padrão comedo $(p=0,0 \mid 9)$.

Observou-se também que todas as amostras teciduais c-erbB-2 positivas exibiam necrose, mas só nove $(32,1 \%)$ das amostras com necrose exprimiam positividade para este oncogene, resultados de acordo com a literatura ${ }^{33,41}(p=0,0066)$.

As relações entre a imunoexpresão do c-erbB-2 e as características nucleares expressas por Lagios ${ }^{26}$ foram publicadas encontrando correlação estatisticamente significante 19,40-43. Resultados comparáveis foram verificados nesta série, na qual oito (40\%) das lesões de grau nuclear 3 exprimiam positividade imunohistoquímica, e somente um dos nove casos c-erbB-2 positivo era de grau nuclear 2; e todos os restantes eram grau $3(p=0,0084)$.

Considerando a classificação de Van Nuys, constatou-se que entre as lesões imunopositivas nenhuma fora classificada como Van Nuys I, uma ( I I, I\%) era Van Nuys 2 e oito $(88,9 \%)$ pertenciam ao grupo Van Nuys 3.

Observou-se que a expressão do c-erbB-2 correlaciona-se com a possibilidade de progressão das lesões epiteliais proliferativas intraductais, não sendo observada em tecidos normais adjacentes às lesões, nas hiperplasias sem atipias e nas neoplasias intraductais classificadas como Van Nuys I, enquanto nos grupos 2 e 3 houve progressivo aumento da expressão.

As correlações entre a expressão dos marcadores biomoleculares, nas lesões proliferativas intraductais da mama, e a predição de riscos de recorrência e progressão para câncer devem ser melhor avaliadas em estudos colaborativos com a participação de múltiplas instituiç̧ões, o que permitirá analisar séries com grande número de casos e com longo seguimento que possam propiciar conclusões definitivas.

\section{ConClusões}

O oncogene c-erbB-2 exibiu correlação significante com as lesões epiteliais proliferativas (LEPI) de maior risco. Não houve expressão nos tecidos adjacentes, nem nas formas mais iniciais de proliferação intraductal como a hiperplasia ductal simples e nas lesões epiteliais proliferativas de baixo grau e baixo risco (Van Nuys I e 2 ).

Por outro lado, a expressão imunohistoquímica do c-erbB-2 correlacionou-se de forma estatisticamente significante com conhecidos fatores prognósticos anatomopatológicos: subtipo comedo, grau nuclear 3 e a presença de necrose.

\section{Conflito de interesse: não há.}

\section{SUMMARY \\ IMMUNOEXPRESSION OF C-ERBB-2 IN INTRADUCTAL PROLIFERATIVE LESIONS OF THE FEMALE BREAST}

OBJECTIVES. Genetic modifications are related to genesis and development of cancer. Neoplasias in various organs express the c-erbB-2 oncogene. In intraductal proliferations of the breast it has been assessed as a risk factor for subsequent development of carcinoma. The cerbB-2 immunoexpression in intraductal epithelial proliferations and the relationship with histopathological characteristics of ductal carcinoma in situ (DCIS) were evaluated.

METHODS. File material from 88 women, which were tissue samples formalin-fixed, paraffin-embedded blocks, was used. Of these 5 I presented with DCIS and 37 with ductal hyperplasia without atypia. Ages of the women ranged from 35 to 76 years.

All cases were reviewed and nuclear grade, presence of necrosis, preponderance of histological subtype and its extension were verified. Specimens were obtained for the c-erB-2 immunohistochemical study of 84 of the women in question.

RESULTS. No expression of the oncogene was verified in the hyperplasias without atypias and in tissues adjacent to all tissue samples. Expression of c-erbB-2 was verified in 9 (19.1\%) of the DCIS $(p=0.000 I)$.

Immunoexpression was not related to the extension of the lesions.

The c-erbB-2 immunoexpression in DCIS was correlated to the histological subtype $(p=0.019)$, necrosis $(p=0.0066)$, nuclear grade $(p=0.0084)$ and $\operatorname{Van}$ Nuys Classification $(p=0.039)$.

Conclusions. Expression of c-erbB-2 was significant in proliferative lesions with risk (DCIS) and was correlated to histopathological characteristics: high nuclear grade, presence of necrosis and comedy subtype. There was no expression in the hyperplasias without atypias and adjacent tissues. [Rev Assoc Med Bras 2004; 50(3): 324-9]

KEY WORDS: Ductal carcinoma "in situ". Intraductal proliferative lesions. C-erbB-2. Prognosis. Breast cancer.

\section{REFERÊNCIAS}

I. Garfinkel L, Boring CC, Heath CW Jr. Changing trends: an overview of breast cancer incidence and morbility. Cancer 1994; 74:222-7.

2. Morrow M, Strom EA, Basset LW, Dershaw DD, Fowble B, Harris JR, et al. Standart for the management of Ductal Carcinoma In Situ of the Breast(DCIS). CA Cancer J Clin 2002; 52:256-76.

3. Broders AC. Carcinoma In Situ contrasted with benign penetrating epithelium. JAMA 1932; 99: 1670-4.

4. Schnitt SJ, Abner A, Gelman R, Connoly JL, Recht A, Duda RB, et al. Relationship between microscopic margins of resection and the risk of local recurrence in the breast cancer patients treated with breast cancerconserving surgery and radiation therapy. Cancer 1994; 74: 1746-5I.

5. Moore E, Magee H, Cayne J, Gorey T, Dervan PA. Widespread chromossomal abnormalities in high grade ductal carcinoma in situ of the breast comparative genomic hybridization study of pure high grade DCIS. J Pathol 1999; 187:403-9. 
6. Schnitt SJ, Harris Jr, Smith BL. Developing a prognostic index for ductal carcinoma in situ of the breast. Cancer 1996; 77:2189-91.

7. Silvertein MJ, editor. Ductal carcinoma in situ of the breast. Baltimore: Willians Wilkins; 1997.

8. Tan $\mathrm{PH}$, Chuah $\mathrm{KI}$, Chiang G, Wong $\mathrm{CY}$, Dong $\mathrm{F}$, Bay $\mathrm{BH}$. Correlation of $\mathrm{p} 53$ and cerbB2 expression and hormonal receptor status with clinicopathologic parameters in ductal carcinoma in situ of the breast. Oncol Rep 2002; 9: $1081-6$.

9. Yarbro JW, Page DL, Fielding LP, Partridge EE, Murphy GP. American Joint Committee On Cancer Prognostic Factors Consensus Conference. Cancer 1999; 86:2436-46.

10. Shih C, Padhy LC, Murray M, Weinberg RA. Transforming genes of carcinomas and neuroblastomas introduced into mouse fibroblast. Nature 1981; 290:261-73.

I I. Allred DC, Clark GM, Molina R, Tandon AK, Schnitt SJ, Gilcherist KW, et al. Over expression of HER-2/Neu and its relationship with other prognostics factors change during the progression of in situ to invasive breast cancer. Hum Pathol 1992; 23:974-9.

12. Barnes DM, Bartkova J, Camplejohn RS, Gullick WJ, Smith PJ, Millis RR, et al. Over expression of the C-Erbb-2 oncoprotein: why does this occur more frequently in ductal carcinoma in situ invasive mammary carcinoma and is this of prognostic significance? Eur J Cancer 1992; 28:648-64.

13. Liu E, Thor A, He M, Barcos M, Britt-Marie L, Benz C. The HER2(C-Erbb-2) oncogene is frequently amplified in "in situ" carcinomas of the breast. Oncogene 1992; 7: 1027-32.

14. Hoque A, SneigeN, Sahin AA, Menter DG, Bacus JW, Hortobagyi GN, et al. Her-2/neu gene amplification in ductal carcinoma in situ of the breast. Cancer Epidemiol Biomarkers Prev 2002; I I:587-90.

15. Stark A, Hulka BS, Joens S, Novatny D, Thor AD, Wold LE, et al. HER-2/Neu amplification in benign breast disease and the risk of subsequent breast cancer. I Clin Oncol 2000; | 8:267-74

16. Brobow LG,Happerfiel D, Gregory WM, Springall R, Millis RR. The classification of ductal carcinoma in situ and it's association with biological markers. Semin Diagn Pathol 1994; 1।:199-207.

17. Somerville JE, Clarke LE, Biggart JD. C-erbB-2 overexpression and histological type of in situ and invasive breast carcinoma. J Clin Pathol 1992; 45: 16-20

18. Ajisaka H, Tsugawa K, Noguch M, Miwa M, Nomomura A. Histological subtypes of ductal carcinoma in situ of the breast. Breast Cancer 2002; 9:55-61.
19. Claus EB, Chu P, Howe CL, Davinson TL, Stern DF, Carter D, et al. Pathobiologic findings in DCIS of the breast: morphologic features, angiogenesis, HER-2/Neu and hormone receptors. Exp Mol Pathol 200I; 70:303-16.

20. DiGiovanna MP, Chu P, Davison TL, Howe $\mathrm{CL}$, Carter D, Claus EB, et al. Active signaling by HER-2/neu in a subpopulation of HER-2/ neu-overexpressing ductal carcinoma in situ: clinicopathological correlates. Cancer Res 2002; 62:667-73.

21. Gandhi A, Holland PA, Knox WF. Evidence of significant apoptosis in poorly differentiated ductal carcinoma in situ of the breast. $\mathrm{Br} J$ Cancer 1998; 78:788-94.

22. Baird RM, Worth A, Hislop G. Reccurence after lumpectomy for comedo-type intraductal carcinoma of the breast. Am J Surg 1990; |59:479-8|.

23. De Luca LA, Vespoli HMDL, Uemura G. Lesões proliferativas da mama. In: BOFF RA. Mastologia aplicada: abordagem multidiciplinar. Caxias do Sul: EDUCS; 200I.p. I0I-32.

24. Recommendations for the reporting of the breast carcinoma.association of directors of anatomic and surgical pathology. Mol Pathol 1996; 1:77-81.

25. Silverstein MJ, Poller DN, Waisman JR, Colburn WJ, Barth A, Gierson ED, et al. Prognostic classification of breast ductal carcinoma in situ. Lancet 1995; 345: I I 54-7.

26. Lagios MD. Duct carcinoma in situ pathology and treatment. Surg Clin North Am 1990; 70:853-7।

27. Consensus Conference Committee. Consensus Conference On The Classification Of Ductal Carcinoma In Situ. Hum Pathol 1997; 28: $|22|-5$

28. Gorczyca W, Markewski M, Kram AL. Immunohistochemical analysis of $\mathrm{BCl}-2$ and P53 expression in breast carcinomas:their correlation with Ki-67 Growth fraction. Virchows Arch 1995; 426:229-33.

29. Fisher RA. Statistical methods for research workers. I Ord ed. New York: Hafner Publishing; 1948.

30. Provenzano E, Hopper JL, Giles GG, Marr G, Venter DJ, Armer JE. Biological markers that predict clinical recurrence in ductal carcinoma in situ of the breast. Eur J Cancer 2003; 39:622-30

31. Mokbel K. Current management of ductal carcinoma in situ of the breast. Int J Clin Oncol 2003; 8: 18-22

32. Perin F, Canzonieri V, Massarut S, Bidoli E, Rossi C, Roncadin M, et al. Immunohistochemical evaluation of multiple biological markers in ductal carcinoma In Situ of the breast. Eur J Cancer 1996; 32A: I| 48-55.
33. Zafrani B, Leroyer A, Fourquet A, Laurent $M$, Trophilme MD, Validire $P$, et al. Mammographically detected ductal in situ carcinoma of the breast analyzed with a new classification.a study of 127 cases: correlation with estrogen and prgesterone receptors, P53 And C-Erbb-2 proteins and proliferaive activity. Semin Diagn Pathol 1994: 1 1:208- 14

34. van De Vijver ML, Peterse JL, Mooi WJ, Wisman P, Lomans J, Dalesio O, et al. Neuprotein overexpression in breast cancer. $\mathrm{N}$ Engl J Med 1988; 319:1239-45.

35. Latta EK, Tjan S, Parkes RK, O'Malley FP. The Role of HER2/neu overexpression/amplification in the progression of ductal carcinoma in situ to invasive carcinoma of the breast. Mod Pathol 2002; 15: 13 |8-25.

36. Chan KC, KnoxWF, Sinha G, Gandhi A, Barr L, Baildam AD, et al. Extent of excision margin width required in breast conserving surgery for ductal carcinoma in situ. Cancer 200 I ; 1:9-16.

37. Ho GH, Calvano JE, Bisogna M, Borgen PI, Rosen PP, Tan LK, et al. In microdissected ductal carcinoma in situ. HER-2/Neu amplification but not P53 mutation, is associated with high nuclear grade and comedo histology. Cancer 2000: 89:21 53-69.

38. Kestin LI, Goldstein NS, Lacerna MD, Balasubramanian M, Morting AA, Rebner M, et al. Factors associated with local recurrence of mammographicaly detected ductal cracinoma in situ in patients given breast-conserving therapy. Cancer 2000; 3:596-607.

39. Bartkova J, Barnes DMm, Millis RR, Gullik WJ. Immunohistochemical demonstration of CErbb-2 protein in mammary ductal carcinoma in situ. Hum Pathol 1990; $21: 1$ 164-7.

40. Bhoola S, Derose PB, Cohen C. Ductal carcinoma in situ of the breast frequency of biomarkers according to histologic subtype. Appl Immunohistochem Mol Morphol 1999; 7:108-15.

4I. Rodrigues NA, Dillon D, Carter D, Parisot N, Haffty BG. Differences in the pathologic and molecular features of intraductal breast carcinoma between younger and older women. Cancer 2003: 97:1393-403.

42. Piccart M, Lohrisch C. An Overview Of HER2. Semin Oncol 200 I; 28:3-11.

43. Walker RA, Dearing SJ, Brown LA. Comparasion of pathological and biological features of symptomatic and mammographically detected ductal carcinoma in situ of the breast. Hum Pathol 1999; 30:943-8.

Artigo recebido: 22/04/2003 Aceito para publicação: 09/03/2004 